MATEC Web of Conferences 24,07004 (2015)

DOI: $10.1051 /$ matecconf/ 20152407004

(C) Owned by the authors, published by EDP Sciences, 2015

\title{
Dynamic investigation of a suspension footbridge using accelerometers and microwave interferometer
}

\author{
Carmelo Gentile $e^{1, a}$ \\ ${ }^{1}$ Politecnico di Milano, Department of Architecture, Built environment and Construction engineering, Milan, Italy
}

\begin{abstract}
The paper presents the main results of the serviceability assessment of a lively suspension footbridge. An ambient vibration test was firstly developed on July 2012 using conventional accelerometers with the objective of identifying the baseline dynamic characteristics of the structure; subsequently, groups of volunteers (up to 32 adults) simulated normal walking and running at different step rates along the deck and the human-induced vibrations were simultaneously measured by accelerometers and microwave interferometer. The deflection responses recorded by the microwave interferometer suggested the exceeding of comfort criteria threshold and this result was confirmed by the acceleration levels directly measured by accelerometers or derived from the (radar) displacement data. Furthermore, a second ambient vibration test was performed in Autumn 2012 using only the microwave interferometer: the natural frequencies of the footbridge generally exhibited not negligible variations, that were conceivably associated to the change of suspension forces induced by temperature, so that special care is suggested in the design of the devices aimed at mitigating the excess of human induced vibrations observed in the footbridge.
\end{abstract}

\section{Introduction}

Microwave remote sensing [1-6] is probably the most recent experimental technique suitable to the non-contact measurement of deflections on large structures, in static or dynamic conditions. The main ideas of the radar-based measurement of deflections are [5]:

1. to employ a radar to take consecutive images of the investigated structure at an appropriate sampling rate. Each radar image represents a distance map of the intensity of radar echoes coming from the reflecting targets: for example, each discontinuity of a structure (such as the "corner zones" corresponding to the intersection of girders and cross-beams in the deck of bridges) represents a good reflecting target and can be identified as a relative maximum of the echo amplitude;

2. to evaluate the displacement of each reflecting target using the phase variation of the back-scattered microwaves associated to that target at different times (microwave interferometry).

The paper addresses the application of conventional accelerometers and microwave remote sensing in the serviceability assessment of a lively pedestrian bridge. The investigated footbridge follows the modern trend to construct light and slender footbridges, accompanied with aesthetic requirements for greater slenderness. The adopted structural solution basically includes: (a) the deck, very slender and about $64.0 \mathrm{~m}$ long; (b) one main spatial system of suspension cables and hangers; (c) two steel frames supporting the suspension cables and the backstays.

The dynamic characteristics of the footbridge were extensively investigated, right after its completion and before the opening, on mid July 2012. More specifically, operational modal testing (with the excitation being mainly provided by micro-tremors and wind) was performed using conventional accelerometers and 14 vibration modes were identified in the frequency range 0 $10 \mathrm{~Hz}$ using different output-only techniques: the frequency of the fundamental mode was $1.03 \mathrm{~Hz}$ and five modes turned out to fall in the frequency range $2-3 \mathrm{~Hz}$. Subsequently, groups of volunteers (up to 32 adults) simulated normal walking and running at different step rates along the deck and the human-induced vibrations were simultaneously measured by accelerometers and microwave interferometer. The deflection responses recorded by the microwave interferometer suggested the exceeding of comfort criteria threshold and this result was confirmed by the acceleration levels derived from the (radar) displacement data. In addition, an excellent comparison was found between velocity and acceleration time series obtained from data simultaneously acquired by accelerometers and radar.

The investigation was completed by a second ambient vibration test, performed in Autumn 2012 using only the microwave interferometer: the critical natural frequencies exhibited not negligible variations, that were conceivably associated to the change of suspension forces induced by temperature, so that special care is suggested in the design of devices aimed at mitigating the excess of human induced vibrations observed in the footbridge.

\footnotetext{
${ }^{\mathrm{a}}$ Corresponding author: carmelo.gentile@polimi.it
} 


\section{Description of the footbridge}

The investigated footbridge (Figs. 1-2) crosses the Serio river in the neighbourhood of the town of Seriate (about $50 \mathrm{~km}$ far from Milan).

The suspended deck is $63.90 \mathrm{~m}$ long and its width varies between $2.5 \mathrm{~m}$ and $5.0 \mathrm{~m}$. The deck consists of timber planks, supported by a grid of steel stringers (exhibiting a rise of $1.3 \mathrm{~m}$, Fig. 1a) and floor beams. The floor beams belong to two classes (Fig. 1b): the main transverse beams (equally spaced at $3.0 \mathrm{~m}$ and characterized by a tapered cross-section) and the secondary IPE 120 cross-beams. The stringers include a couple of IPE 330 edge beams and a central girder with a hollow circular section $(\varnothing=298.5 \mathrm{~mm})$. All the longitudinal beams are connected to the main transverse beams through bolted connections capable of restoring the continuity, whereas the connection between the secondary beams and the edge beams allows the secondary beams to rotate around the edge beam axis. The ends of the main floor beams are crossed by a stabilizing cable (Fig. 1b), whose sliding in the longitudinal direction is permitted by the interposition of a polymeric layer between the contact surfaces. The deck is completed by timber planks, $5.0 \mathrm{~cm}$ thick, providing the walking surface and support for the pedestrians.

The suspension system supporting the deck consists of:

- 4 steel pylons, exhibiting a slight slope with respect to the vertical plane and arranged to constitute two Ashaped portal frames. The main suspension cables and the backstays are connected to the top of the pylons as it is shown in Figs. 1-2;

- 2 main suspension cables, of $60 \mathrm{~mm}$ diameter, supporting the deck through 42 hangers of $16 \mathrm{~mm}$ diameter;

- 4 backstays, of $60 \mathrm{~mm}$ diameter, linking the pylons to the ground;
- 2 lateral stabilizing cables, of $40 \mathrm{~mm}$ diameter and with opposing curvature (Fig. 1b).

As shown in Figs. 1-2, the suspension system is not symmetric about the vertical plane crossing the longitudinal bridge axis: each of the two hangers supporting the opposite ends of the same cross-beam has different length and the main cables do not follow the same parabolic shape.

(a)

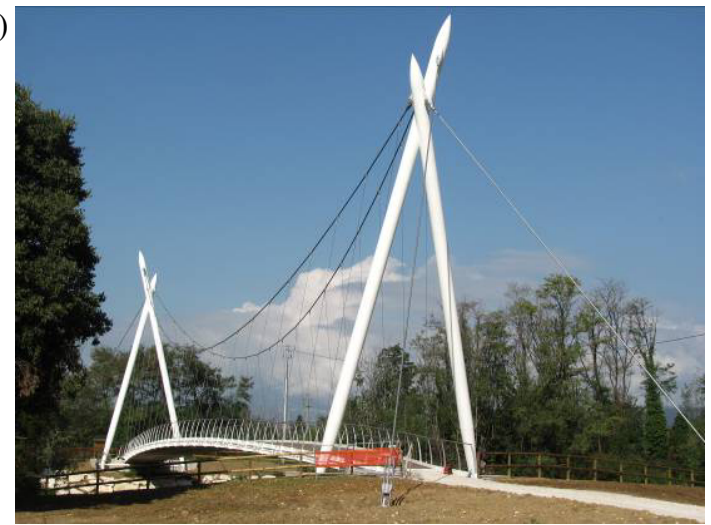

(b)

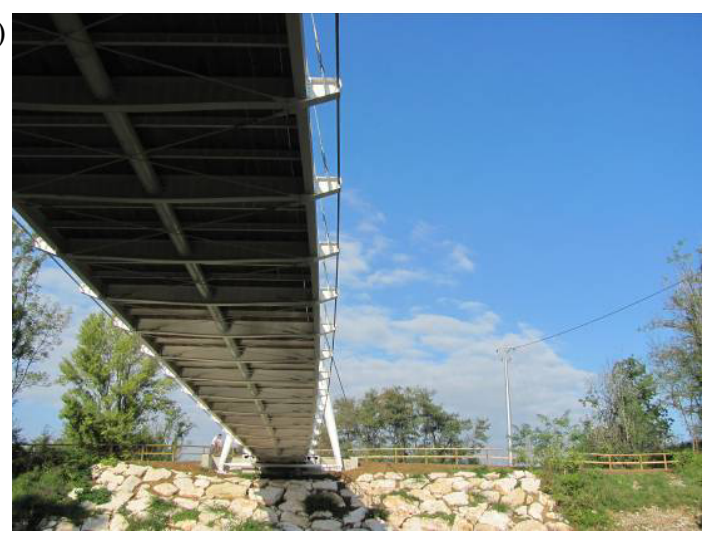

Figure 1. (a) General view of the investigated footbridge; (b) Underside view of the deck

(a)

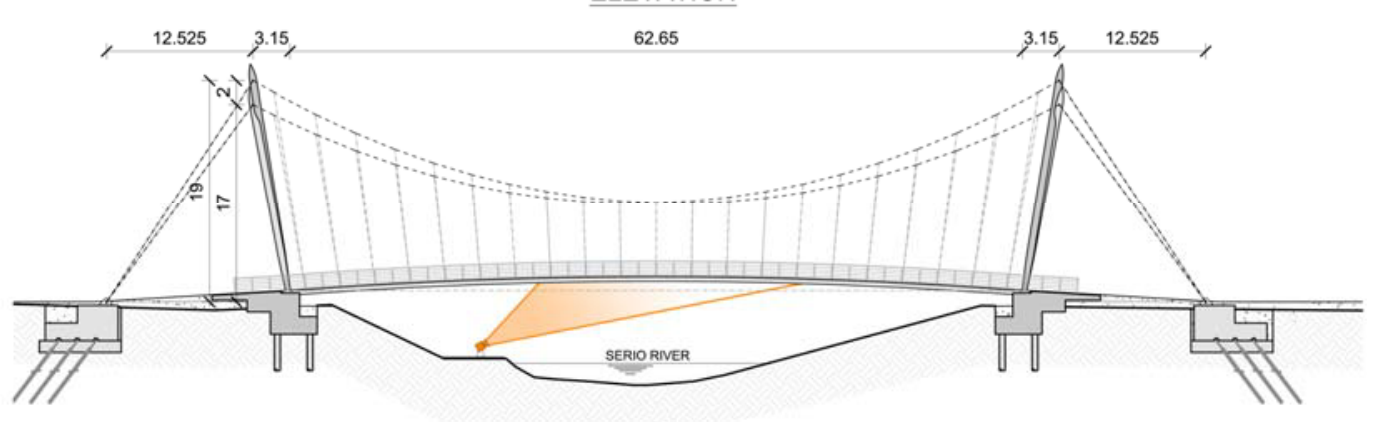

(b)

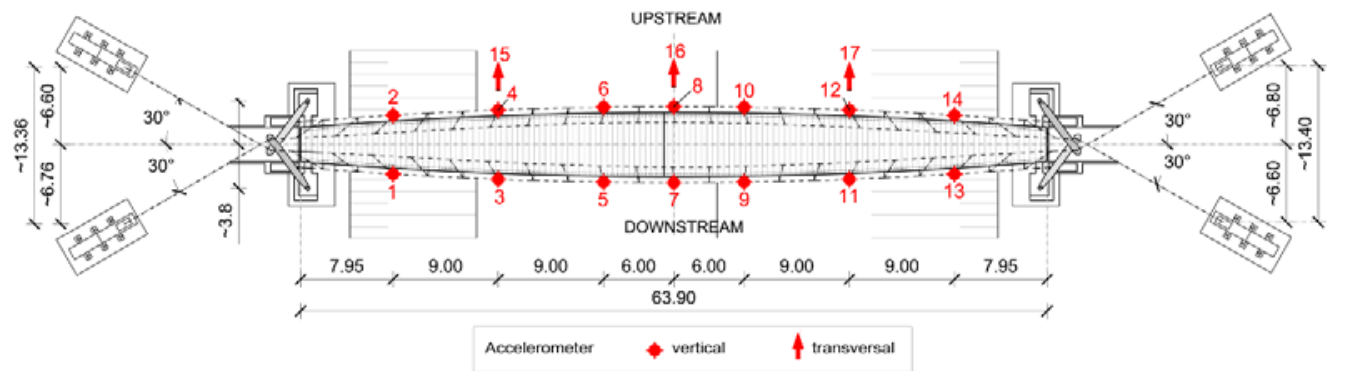

Figure 2. (a) Elevation of the footbridge (dimensions in $\mathrm{m}$ ) and radar position during the field tests; (b) Plan of the footbridge (dimensions in $\mathrm{m}$ ) and accelerometers layout 


\section{Experimental procedures and testing equipments}

The dynamic characteristics of the footbridge were extensively investigated, right after its completion and before the opening, on mid July 2012. More specifically, an ambient vibration test (AVT) was firstly developed with the objective of identifying the baseline dynamic characteristics (i.e. natural frequencies, mode shapes and damping ratios) of the structure; subsequently, walking and running tests were carried out to verify that the human-induced vertical and horizontal accelerations were limited to acceptable values. In the walking tests, the structural response associated to groups of 4, 8, 16 and 32 pedestrians crossing the bridge were measured, whereas the running of only 4 joggers was carried out.

During both the AVT and the walking and running tests, the response of the footbridge was measured at 17 selected points in a single set-up (Fig. 2b), using a 24channel data acquisition system (24-bit resolution, 102 $\mathrm{dB}$ dynamic range and anti-aliasing filters) with uni-axial WR 731A piezoelectric accelerometers. Each WR 731A sensor, capable to measure accelerations of $\pm 0.50 \mathrm{~g}$ with a nominal sensitivity of $10 \mathrm{~V} / \mathrm{g}$, was connected with a short cable $(1 \mathrm{~m})$ to a WR $\mathrm{P} 31$ power unit/amplifier providing the constant current needed to power the accelerometer's internal amplifier, signal amplification and selective filtering.

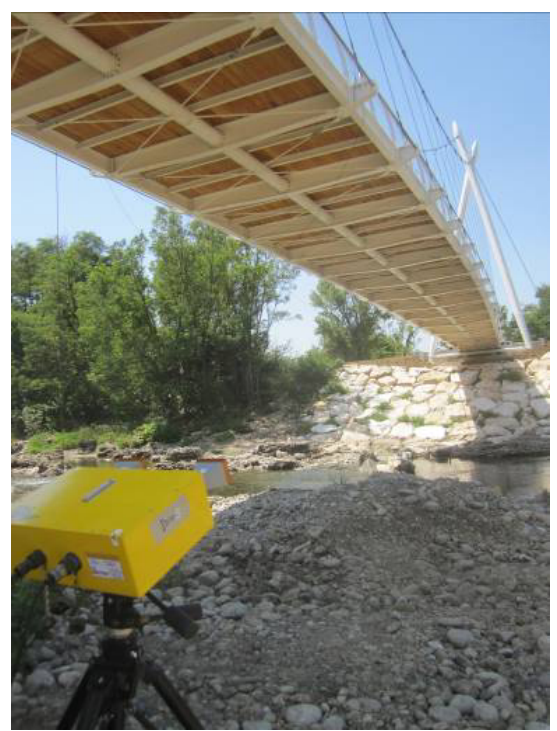

Figure 3. View of the radar sensor (IDS, model IBIS-S)

During the walking and running tests, also a microwave interferometer (Fig. 3) was used. The radar sensor used in this work is an industrially engineered microwave interferometer (IDS, IBIS-S system). Only few information about the main characteristics of the radar sensor will be summarized herein. The interested reader is referred to [3-5] for a more extensive background on the radar techniques adopted in the sensor.

The microwave interferometer consists of a sensor module, a control PC and a power supply unit. The sensor unit is a coherent radar (i.e. a radar preserving the phase information of the received signal) generating, transmitting and receiving the electromagnetic waves. Electromagnetic signals are emitted at a central frequency of $17.2 \mathrm{GHz}$ with a maximum bandwidth of $300 \mathrm{MHz}$, so that the radar is classified as $\mathrm{K}_{\mathrm{u}}$-band, according to the standard radar-frequency letter-band nomenclature from IEEE Standard 521-1984. The sensor unit is installed on a tripod equipped with a 3D rotating head (Fig. 3), allowing the sensor to be orientated in the desired directions. Data control and acquisition are completely managed through a laptop via a standard USB interface; the control PC is provided with the software described in [4] for the system management, data storage, basic signal processing and preliminary view of the results in real time.

The main information provided by the microwave interferometer is the synthetic image of the scenario and the time histories of the points in the scenario that are characterized by a good electromagnetic reflectivity.

The synthetic image of the scenario, or range profile, is simply a 1-D map of the intensity of the received radar echoes in function of the distance of the target points generating the echoes themselves. The scenario illuminated by the radar beam is sampled at distance intervals corresponding to the range resolution. (i.e. the minimum separation that can be detected along the radar's line of sight). The peaks in the range profile identify the position/range of the targets characterized by a good electromagnetic reflectivity. The phase differences between (the corresponding peaks of) two range profiles acquired at different time instants are proportional to the motion of the targets along the radar line of sight.

In the tests of the footbridge, the radar sensor was installed under the deck (Fig. 2a) in eccentric position (exactly below the instrumented point denoted as 3 in Fig. 2b) and inclined $25^{\circ}$ upward (Fig. 3). Fig. 2a schematically shows the position of the radar on site as well as the angle of transmission covered by the main lobe of standard antenna in the vertical plane, with all the points inside the shadowed area of Fig. $2 \mathrm{a}$ being observable from the sensor.

It should be noticed that a second AVT was performed on October 2012 using only the interferometer and adopting the same acquisition geometry illustrated in Figs. 2a and 3.

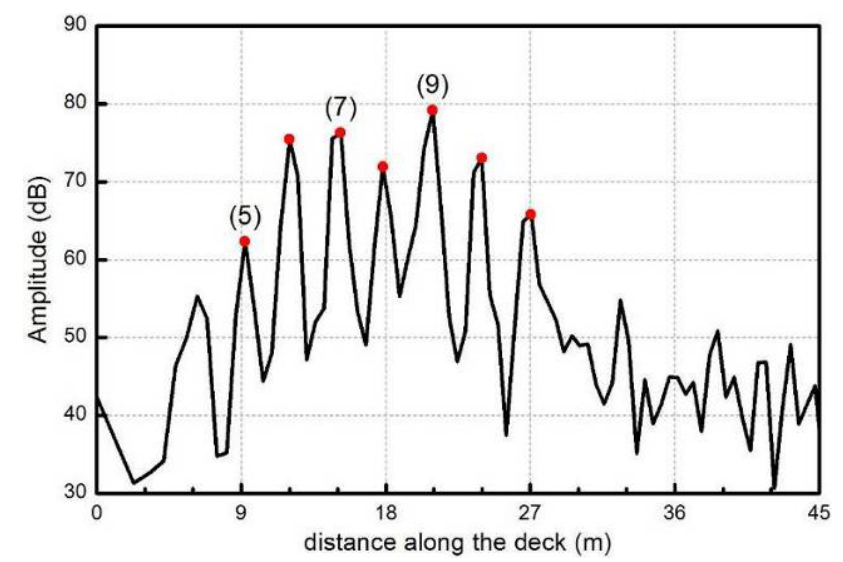

Figure 4. Radar range profile of the test scenario provided by the underside of the footbridge deck 
The range profile obtained from the adopted radar position is illustrated in Fig. 4 and exhibits well defined peaks, clearly identifying the position of 7 equally spaced transverse beams, including the ones hosting the measurement points (accelerometers) denoted as 5, 7 and 9 in Fig. 2 b.

The main technical characteristics of the IBIS-S sensor are the following:

- maximum range (distance) resolution: $0.50 \mathrm{~m}$;

- maximum sampling frequency: $200 \mathrm{~Hz}$;

- maximum operational distance: $\quad 500 \mathrm{~m}$;

- displacement accuracy: $<0.02 \mathrm{~mm}$.

It is worth underlining that the microwave interferometer has only 1-D imaging capabilities, i.e. different targets can be unambiguously detected if they are placed at different distances from the radar; hence, measurement errors may arise from the multiplicity of contributions coming from different points placed at the same distance from the radar [2-4]. Furthermore, the sensor measures displacement along the line of sight (LOS) only so that the evaluation of actual deflections requires the prior knowledge of the direction of motion.

\section{Dynamic characteristics of the bridge}

As previously pointed out, two AVTs were performed on the footbridge.

In the first test, only the acceleration responses mainly induced by micro-tremors and wind were collected and time series of $7200 \mathrm{~s}$ (corresponding to more than 7000 times the fundamental period of the footbridge) were processed using the Frequency Domain Decomposition (FDD) [7] and the covariance-driven Stochastic Subspace

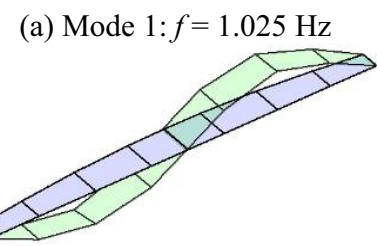

(b) Mode 2: $f=1.475 \mathrm{~Hz}$

(e) Mode $5: f=2.168 \mathrm{~Hz}$

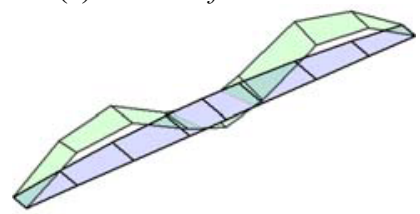

(i) Mode 9: $f=4.121 \mathrm{~Hz}$
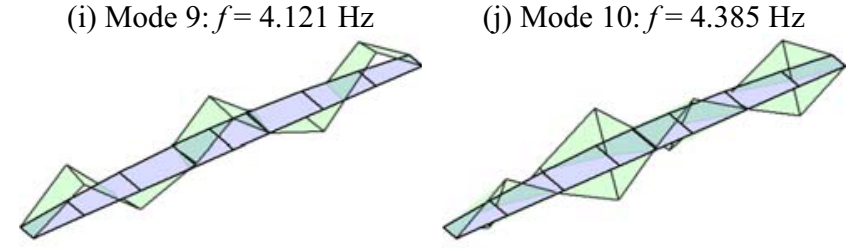

(m) Mode 13: $f=7.217 \mathrm{~Hz}$

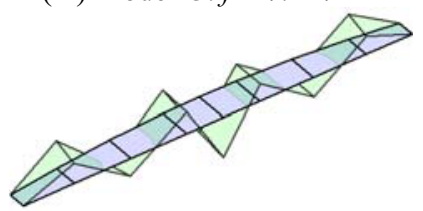

Identification (SSI-Cov) [8] techniques in order to obtain estimates of natural frequencies, damping ratios and mode shapes.

In the second test, only the deflections induced by the ambient excitations were measured using the microwave interferometer and time series of $3600 \mathrm{~s}$ were acquired. The modal parameters were identified mainly by applying the FDD method.

It worth mentioning that the two tests were carried out on 18 July 2012 and 3 October 2012, respectively, with the difference in the air temperature being about $12^{\circ} \mathrm{C}$.

The vibration modes identified in the first dynamic test using the FDD algorithm available in the ARTeMIS software [9] are shown in Fig. 5. The inspection of Fig. 5 highlights that:

a) the frequency of the fundamental mode is about 1.03

$\mathrm{Hz}$ and the corresponding mode shapes involves antisymmetric vertical bending of the deck;

b) almost all modes can be classified as dominant bending or dominant torsion, whereas only one mode (Fig. 5d) exhibits coupling between vertical and transversal components;

c) five vibration modes turned out to fall in the critical frequency interval of 1.9-3.0 Hz.

Very similar results were obtained using the SSI-Cov algorithm developed in [10]. For example, Table 1 summarizes the results obtained by applying the FDD and the SSI-Cov methods through: (1) the natural frequencies $\left(f_{\text {FDD }}\right)$ identified by the FDD technique; $(2)$ the natural frequencies $\left(f_{\mathrm{SSI}}\right)$ identified by the SSI-Cov method and (3) the average and standard deviation values of the modal damping ratios $\left(\zeta_{\text {SSI }}, \sigma_{\zeta}\right)$ estimated by the SSI-Cov method.

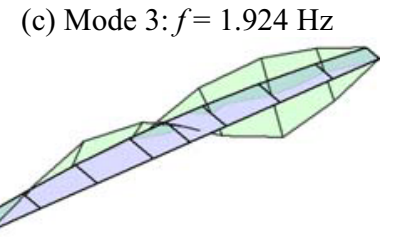

(g) Mode 7: $f=2.861 \mathrm{~Hz}$

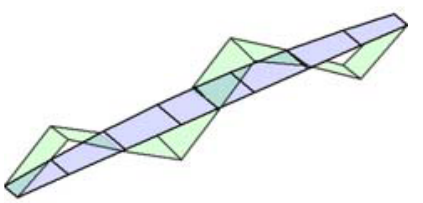

(k) Mode 11: $f=5.645 \mathrm{~Hz}$

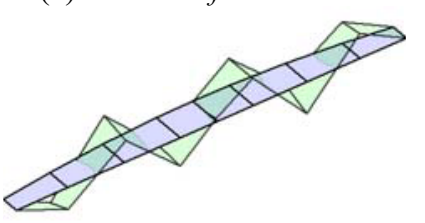

(n) Mode 14: $f=7.490 \mathrm{~Hz}$

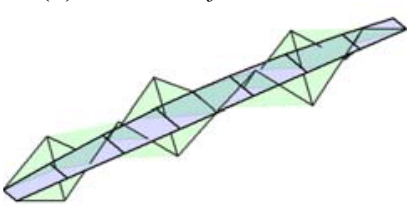

(d) Mode 4: $f=1.953 \mathrm{~Hz}$

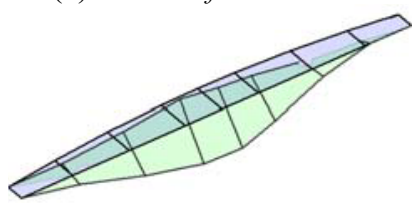

(h) Mode $8: f=3.691 \mathrm{~Hz}$

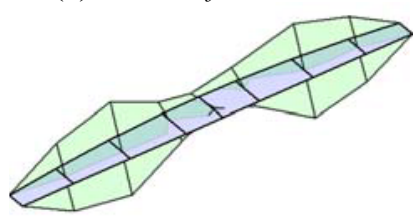

(1) Mode 12: $f=6.006 \mathrm{~Hz}$

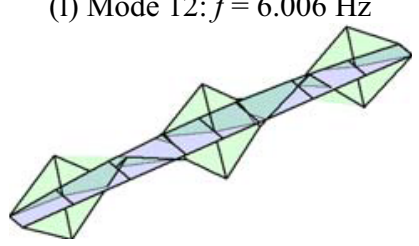

Figure 5. Vibration modes identified from accelerometer data (FDD, 18/07/2012) 
Table 1. Summary of the modal parameters identified by FDD and SSI-Cov methods (accelerometer data, 18/07/2012)

\begin{tabular}{cccccc}
\hline \multirow{2}{*}{$\begin{array}{c}\text { Mode } \\
\text { N. }\end{array}$} & \multirow{2}{*}{ Mode Shape } & FDD & \multicolumn{3}{c}{ SSI-Cov } \\
\cline { 3 - 6 } & $f_{\text {FDD }}(\mathrm{Hz})$ & $f_{\text {SSI }}(\mathrm{Hz})$ & $\zeta_{\text {SSI }}(\%)$ & $\sigma_{\zeta}(\%)$ \\
\hline \hline 1 & Vertical / bending & 1.025 & 1.021 & 1.17 & 0.040 \\
2 & Vertical / bending & 1.475 & 1.473 & 1.44 & 0.039 \\
3 & Vertical / torsion & 1.924 & 1.923 & 0.91 & 0.030 \\
4 & Vertical / Transv. & 1.953 & 1.949 & 1.02 & 0.014 \\
5 & Vertical / bending & 2.168 & 2.168 & 0.72 & 0.035 \\
6 & Vertical / torsion & 2.754 & 2.755 & 1.09 & 0.006 \\
7 & Vertical / bending & 2.861 & 2.866 & 0.55 & 0.008 \\
8 & Vertical / torsion & 3.691 & 3.694 & 0.82 & 0.011 \\
9 & Vertical / bending & 4.121 & 4.136 & 1.20 & 0.091 \\
10 & Vertical / torsion & 4.385 & 4.380 & 0.91 & 0.123 \\
11 & Vertical / bending & 5.645 & 5.637 & 0.62 & 0.004 \\
12 & Vertical / torsion & 6.006 & 6.006 & 0.60 & 0.015 \\
13 & Vertical / bending & 7.217 & 7.218 & 0.74 & 0.019 \\
14 & Vertical / torsion & 7.490 & 7.495 & 0.78 & 0.006 \\
\hline
\end{tabular}

It is worth mentioning that the evaluation of damping ratios using the SSI-Cov method was performed for increasing values of the time delay parameter $i$, in order to obtain accurate estimates of the damping ratios, especially for the vibration modes in the critical range of frequency 1.9-3.0 Hz. The estimates of damping ratio exhibited an excellent stability for $i>120$ and the corresponding standard deviations, listed in Table 1, generally did not exceed $0.04 \%$.

(a)

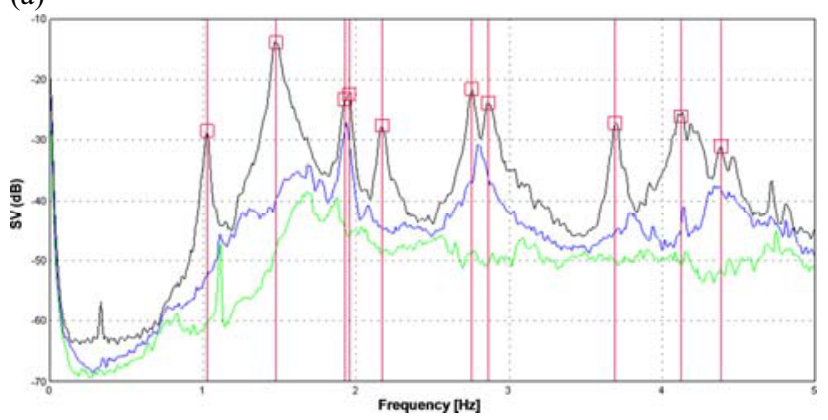

(b)

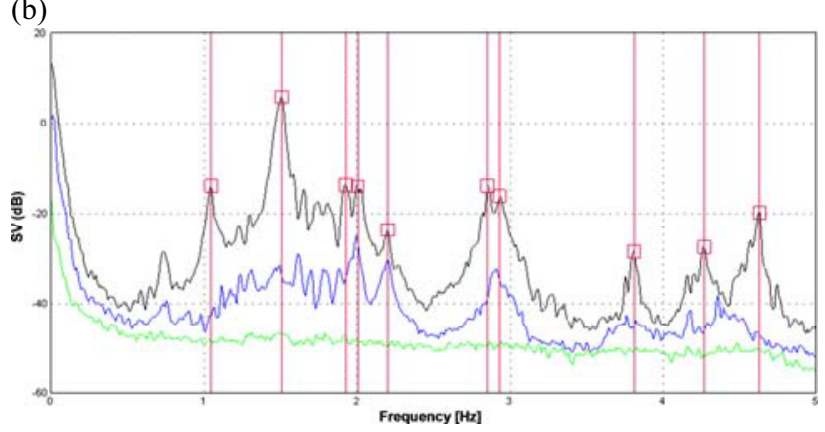

Figure 6. Singular value lines (SV) and identification of natural frequencies: (a) 18/07/2012, accelerometer data; (b) $03 / 10 / 2012$, displacement (radar) data

It is interesting to compare the modal parameters estimated from the accelerometer data collected in July 2012 and the displacement (radar) data recorded in October 2012. The comparison in terms of natural frequencies is summarized in Figs. 6a (accelerometer data, 18/07/2012) and 6b (displacement data,
03/10/2012): the frequency of spectral peaks in the first singular value (SV) line related to the second test (Fig. 6b) exhibits a clear increase with respect to the first test (Fig. 6a) and only the frequency of the fourth peak (corresponding to the only mode characterized by significant transversal components, Fig. 5d) remains practically unchanged. The observed decrease of modal frequencies with increased temperature is conceivably associated to the change of suspension forces induced by temperature.

\section{Assessment of pedestrian effects using accelerometer and microwave interferometer}

The analysis of the identified frequencies and characteristics of mode shapes, summarized in Fig. 5 and Table 1, highlighted the existence of 5 natural frequencies in the range 1.9-3.0 Hz, critical for the vertical vibrations excited by groups of pedestrians walking or running. Hence, in order to assess the levels of vibration, various loading scenarios were defined and simulated on site with the help of groups of volunteers (up to 32 adults).

Different loading scenarios were considered: (a) walking of groups of 4, 8, 16 and 32 pedestrians; (b) running of 4 joggers.

Figs. 7 and 8 exemplify the human-induced vibrations in terms of vertical accelerations and vertical deflections measured by conventional sensors and microwave remote sensing during the walking and running loading scenarios respectively. that:

The inspection of Fig. 7 (walking scenario) reveals

a) even for the walking of small groups of pedestrians (4-8 pedestrians), the recorded accelerations and displacements were relatively high in the vertical direction;

b) increasing the number of pedestrians from 4-8 to 1632 does not produce a significant increase of the maximum vertical acceleration as it was difficult to 
(a) Measurement point 5
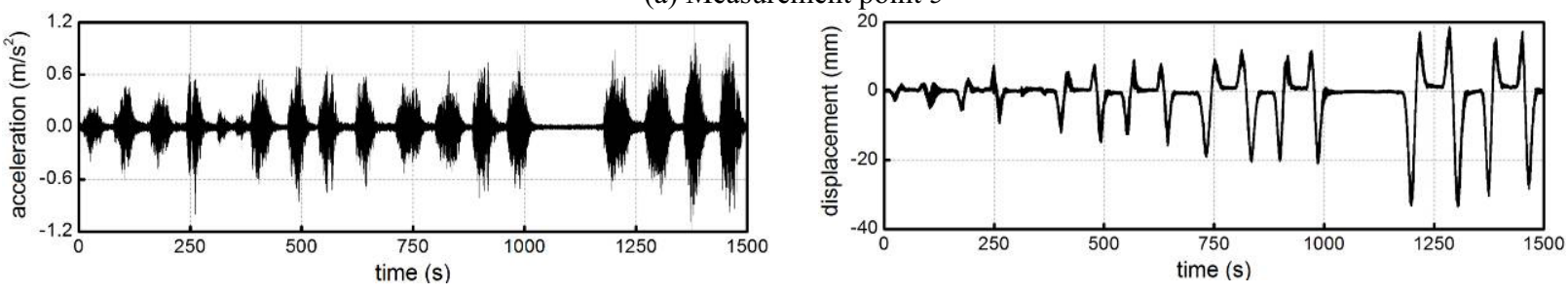

(b) Measurement point 7
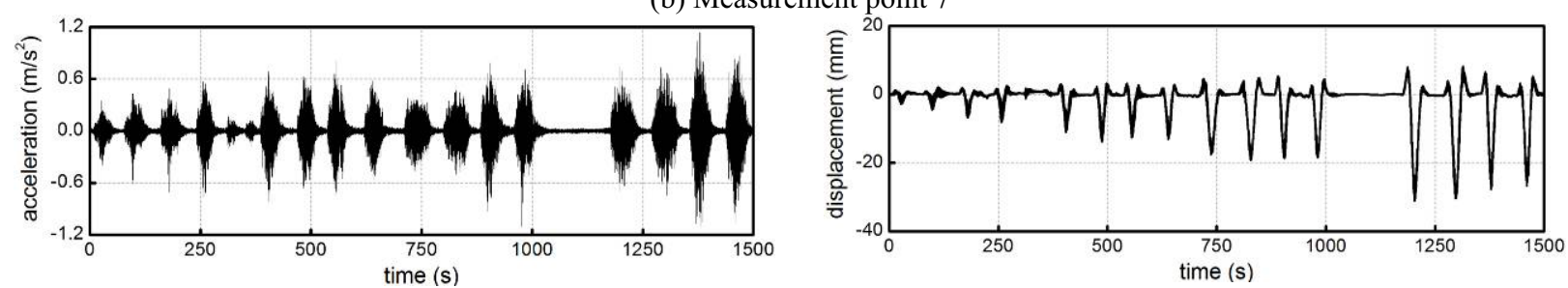

(c) Measurement point 9
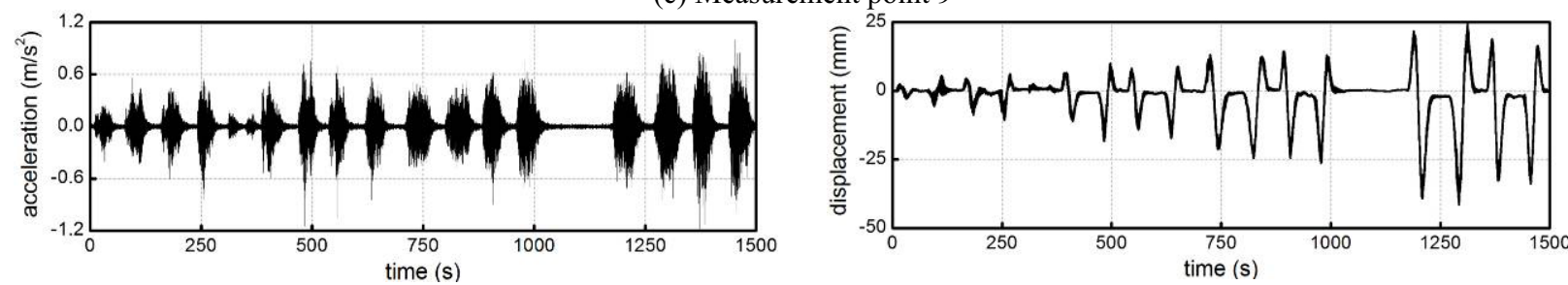

Figure 7. Time series recorded by accelerometers and radar at different points of the deck during the walking tests

Accelerometer Data

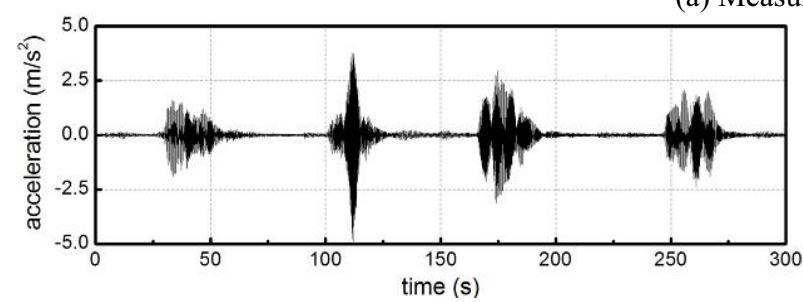

(b) Measurement point 7

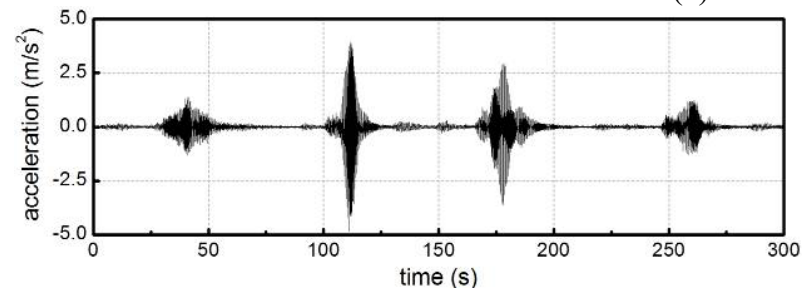

(c) Measurement point 9
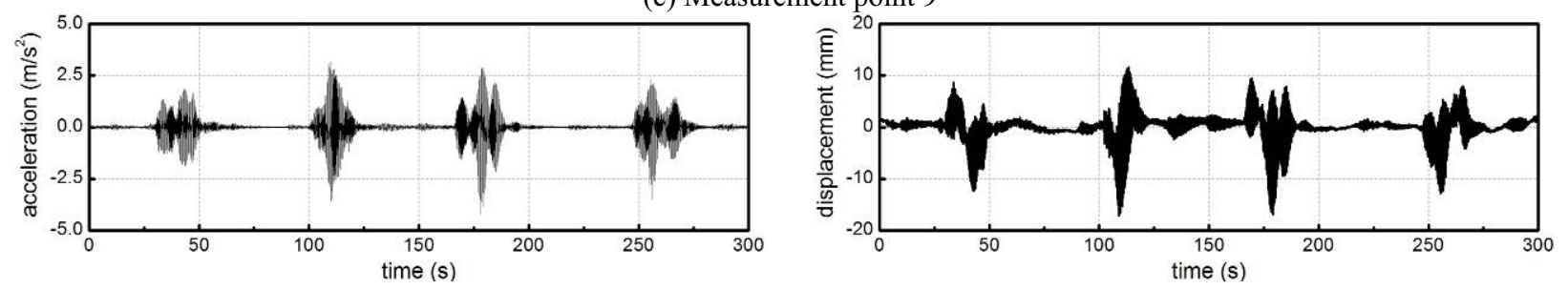

Figure 8. Time series recorded by accelerometers and radar at different points of the deck during the running tests

to ensure the synchronisation between the pedestrians as the number of pedestrians increases;

c) on the contrary, the displacements measured by the radar sensor clearly increase with the increased number of pedestrians.

Fig. 8 (running scenario) highlights that the maximum recorded acceleration induced by the running of 4 joggers is extremely high $(0.48 \mathrm{~g})$ and significantly exceeds the limit acceleration targets suggested in international standards; furthermore, the vertical displacements are relatively high (around $20 \mathrm{~mm}$ ) as well and exhibit clear dynamic amplification.

It is especially interesting to compare: (a) the acceleration and velocity obtained by deriving the 
deflection time series measured by the radar and (b) the corresponding time series directly measured by the accelerometers or obtained by integrating the measured accelerations. The comparison is exemplified in Fig. 9 for one running scenario (Fig. 8): it should be noticed that the velocity and acceleration time histories obtained from the two different sensors are often almost undistinguishable. Hence, the radar technique turned out to be very effective for the use in the serviceability assessment of footbridges because, also depending on the narrowband frequency content of running and walking excitation, the measurement provides with accurate and complete information in terms of displacement, velocity and acceleration. The main limitation in the presented investigation is related to the relatively limited extension of the deck area illuminated by the radar beam, as shown in Fig. 2a; on the other hand, Fig. $2 \mathrm{a}$ also shows that this area tends to significantly and quickly increase with the increased vertical distance between the sensor and the underside of the deck.
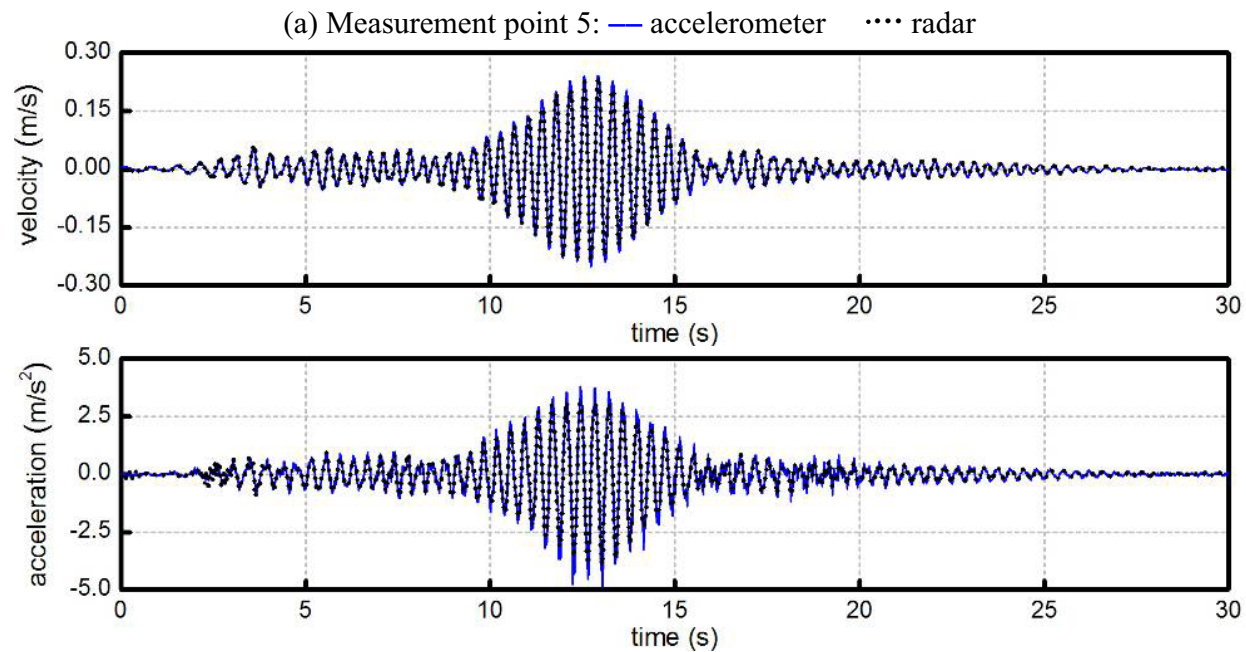

(b) Measurement point 7: - accelerometer $\cdots \cdot$ radar
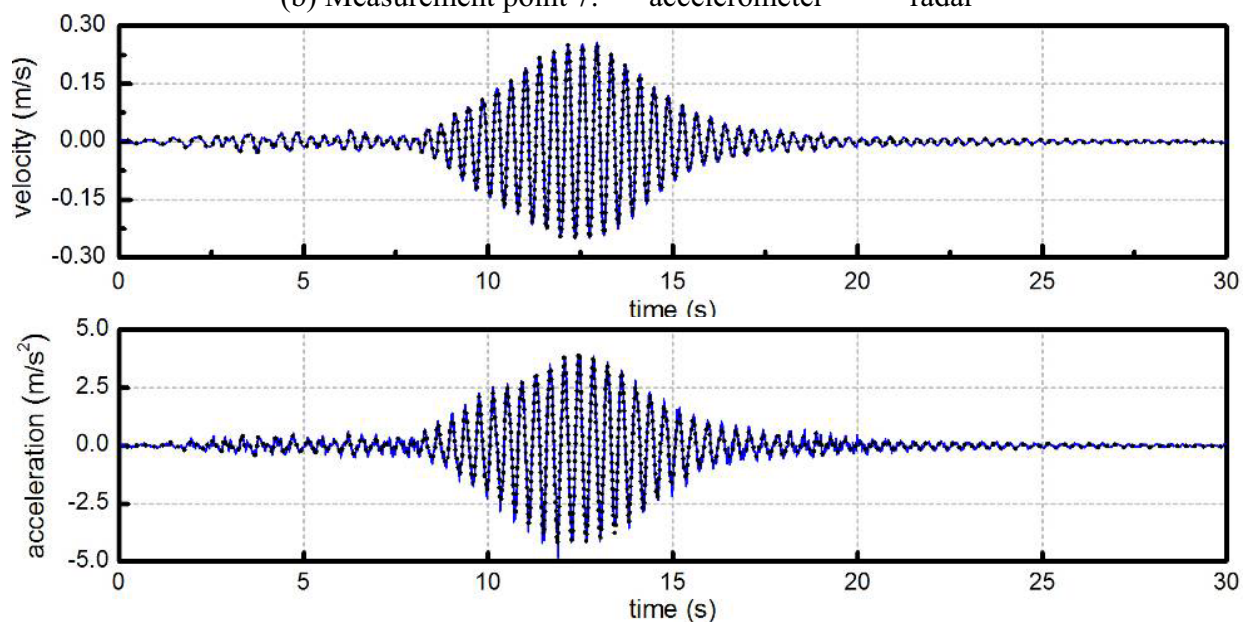

(c) Measurement point 9: - accelerometer $\cdots \cdot$ radar
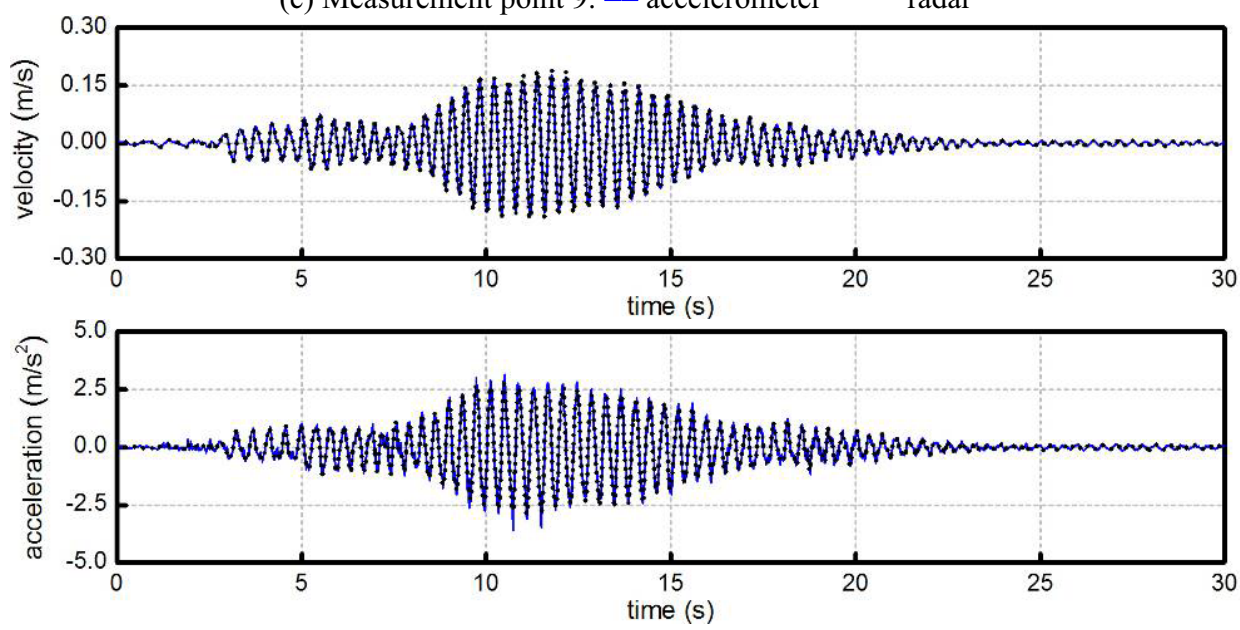

Figure 9. Typical comparison between the time series collected by conventional accelerometers and radar 


\section{Conclusions}

The paper summarizes the main results of the dynamic tests performed on a lively suspension footbridge. The experimental investigation involved both operational modal testing and the definition of various loading scenarios (walking and running) that were simulated on site with the help of groups of volunteers (up to 32 adults). Conventional accelerometers and microwave remote sensing were adopted to measure the response of the footbridge to ambient and human excitation. More specifically: (a) an ambient vibration test was firstly performed using only conventional accelerometers; (b) subsequently, walking and running tests were carried out and the human-induced vibrations were measured by accelerometers and microwave interferometer; (c) a second ambient vibration test was performed about 2 months later using only the microwave interferometer.

The following main conclusions can be drawn:

1. Within the frequency range $0-8 \mathrm{~Hz}, 14$ vibration modes were successfully identified from accelerometer data in operational conditions, using the FDD and the SSICov techniques and a good agreement was generally found between the two methods in terms of both natural frequencies and mode shapes;

2. 5 vibration modes turned out to fall in the critical frequency interval of 1.9-3.0 Hz;

3. Similar dynamic characteristics were successfully identified using the radar interferometer in the second test at a lower air temperature but the identified natural frequencies turned out to increase. This effect (frequency increase with decreased temperature) is conceivably associated to the change of suspension forces induced by temperature;

4. Microwave remote sensing turned out to provide accurate information on the human-induced vibrations, in terms of displacement, velocity and acceleration, although these information were limited to few reflecting points of the deck.

\section{Acknowledgements}

The support of IDS (Ingegneria Dei Sistemi, Pisa, Italy) in supplying the IBIS-S radar sensor employed in the tests is gratefully acknowledged.

The author would like to thank: (a) G. Marrongelli for the valuable help in the operational modal analysis of accelerometer data; (b) M. Antico and M. Cucchi (VIBLAB, Politecnico di Milano) for the assistance in conducting the field tests.

\section{References}

1. M. Pieraccini, M. Fratini, F. Parrini, G. Macaluso, C. Atzeni, Electron. Lett. 40(14), 907-908 (2004)

2. C. Gentile, G. Bernardini, NDT \& E Int. 41(7), 544553 (2008)

3. C. Gentile, G. Bernardini, J. Struct. Infrastruct. Eng. 6(5), 521-534 (2010)

4. F. Coppi F., C. Gentile, P. Ricci, Proc. of the $9^{\text {th }}$ Int. Conf. on Vibration Measurements by Laser and
Noncontact Techniques, AIVELA 2010: 190-201 (2010).

5. C. Gentile, Proc. of the $8^{\text {th }}$ Int. Conf. on Structural Dynamics, EURODYN 2011: 92-103 (2011)

6. G. Luzi, M. Crosetto, M. Cuevas-González, (Barcelona). Mech. Syst. Signal Pr. 49: 234-248 (2014)

7. R. Brincker, L.M. Zhang, P. Andersen, Proc. Of the $18^{\text {th }}$ Int. Modal Analysis Conf., IMAC-XVIII: 625630 (2000)

8. B. Peeters, G. De Roeck, Mech. Syst. Signal Pr. 13(6): 855-78 (1999)

9. SVS, http://www.svibs.com/, ARTeMIS Extractor 2011

10. G. Marrongelli G. Methods and algorithms of Operational Modal Analysis: theory and applications to R.C. bridges (MSc Thesis, Universisty of L'Aquila, 2014) 